\title{
Mental health law in Peru: work in progress
}

\author{
David Jimenez, ${ }^{1}$ Christina Alejandrina Eguiguren, ${ }^{2}$ Dominic Dougall, ${ }^{3}$ \\ Bartłomiej Pliszka ${ }^{4}$ and Ian Hall ${ }^{5}$
}

'Specialty Registrar in Psychiatry, South London and Maudsley NHS Foundation Trust, London, UK, and President, SUD-World Project, email drdavidjimenezleiva@ email drdavidil.com
gmail

${ }^{2}$ Medical Director, Hospital Larco Herrera, Lima, Peru

${ }^{3}$ Consultant Psychiatrist, East London NHS Foundation Trust, London, UK, and SUD-World

"Research Assistant, South London and Maudsley NHS Foundation Trust, London, UK and SUD-World Project

${ }^{5}$ Consultant Psychiatrist, East London NHS Foundation Trust, London, UK, and Chair SUDWorld Project
Mental health law in Peru is developing. The Peruvian Constitution enshrines important human rights principles in relation to people with mental health problems but the enactment of such principles into national legislation is very patchy. This means that people with mental health problems, especially those admitted to hospital, may not receive optimum care and may be at risk of having their human rights breached. In this article we consider how far the current national legislation meets these constitutional rights and what the legislation that is in development may ultimately achieve.

Mental health law in Peru is developing in the context of changing mental health services. Since 1920, the Peruvian Constitution has enshrined important human rights principles in relation to people with mental health problems, but the enactment of such principles into national legislation is currently patchy. This means that people with mental health problems, especially those admitted to hospital, may not receive optimum care and may be at risk of having their human rights breached. In that regard, Peru is a signatory to the 1978 American Convention on Human Rights.

Here we consider how far the current national legislation meets these constitutional rights and what the legislation that is in development may ultimately achieve.

\section{Overview of legislative developments}

Peru does not have a dedicated mental health law at present. Legislation relevant to mental health falls under the General Health Law (Law 26842 of 1979). Article 11 of this law states that every individual has the right to the prevention of mental illness, recovery, rehabilitation and promotion of his or her mental health. The article includes drug and alcohol addictions and domestic violence as mental health problems, in addition to psychiatric disorders. The article makes no provision in relation to involuntary admission to hospital.

In July 2011 a new law (number 29675 of that year) was proposed to amend article 11 of the General Health Law, with the main aim of regulating involuntary admissions. However, it was not adopted as it did not comply with the American Convention on Human Rights.

In 2012 the National Committee of Mental Health attempted to draft a new law (number 29889) again to amend article 11. The law aimed to regulate involuntary admissions, reduce institutionalisation and ensure compliance with human rights. Additional aims included improved prevention and the promotion of mental health. Measures to require local authorities to fund and implement community services were also included. Two types of community home were proposed:

- casas de medio camino - rehabilitation care homes promoting the social integration of psychiatric patients

- hogares protegidos - for psychiatric patients without families, requiring continuing care but not assessment or treatment in hospital.

Drafts of Law 29889 have been sent back for revision several times by the legislature (most recently in January 2013), because of ongoing human rights concerns.

On 24 December 2012, Congress passed Law 29973, the General Law on People with Disabilities, which reiterates the promotion of mental health and recognises diversity and equality.

\section{Definition of mental disorder}

Neither the General Health Law nor the General Law on People with Disabilities has a clear definition of mental disorder; as noted above, article 11 of the General Health Law considers alcoholism, drug addiction and domestic violence to be mental health problems that warrant mental healthcare (voluntary and involuntary), prevention, promotion and rehabilitation. Nonetheless, the DSM-IV criteria for mental disorders (American Psychiatric Association, 1994) are widely applied in Peru.

\section{Grounds for detention}

The General Health Law has no provisions for the regulation of involuntary admissions. However, the Constitution of the Republic of Peru does enshrine certain rights for people with mental health problems. Under article 2 every individual (whether or not he or she has a disability) has the right to liberty, deprivation of which is permitted only if an individual has broken a law. Similarly, the 1978 American Convention on Human Rights establishes that no one should be deprived of their liberty, except under conditions legitimately determined by pertinent laws.

In relation to the admission of psychiatric patients, the Constitution protects the rights of patients, requires the delivery of high standards of mental healthcare and distinguishes between voluntary and involuntary admission. 
According to article 15 of the Constitution, when a person requires treatment in a psychiatric institution, measures should be put in place to help avoid an involuntary admission. Voluntarily admitted patients must authorise their treatment, and they have the right to leave the psychiatric institution at any time, unless there are sufficient grounds to justify an involuntary detention.

Article 16 determines that a patient can be detained involuntarily only when a physician establishes that this person suffers from a mental disorder and considers that:

- due to the mental disorder, there is an immediate or imminent risk to the patient or others

- the mental disorder is severe and the patient's capacity is affected

- there is the prospect of a significant deterioration in the patient's clinical condition

- the appropriate treatment would not otherwise be given.

Treatment should nonetheless be provided according to the principles of least restriction.

Article 16 also states that involuntary admissions should be time limited and determined by national legislation, and that an independent body should scrutinise the admission.

Article 17 states that patients have the right to ask an independent body to question decisions involving their treatment, and that their admission to the psychiatric institution will be periodically reviewed by this independent body.

Despite the Constitution of the Republic requiring the protection of the rights of psychiatric patients and requiring national legislation to govern this, there are as yet no laws enacted that specifically regulate voluntary and involuntary admission. In addition, an independent body has not been established to review involuntary admissions.

\section{The role of the family and informed consent to treatment}

General Health Law 26842 states that people can give informed consent only if they have capacity, have been given information prior to procedures and have not been coerced.

For those who lack capacity, decisions about their care should be made by their family, or in their absence by the appropriately allocated curator (advocate) provided by the Ministry of Justice. The family or curators can discharge patients from psychiatric institutions provided the medical team agrees, but there is no clear legal framework for this, and medical decisions overrule the role of the family or curator when the required treatment is deemed necessary and urgent, to prevent an immediate and imminent risk to the patient or others.

Any treatment can be given to those patients who are admitted involuntarily, without the need to obtain informed consent or other safeguards.
This includes mechanical restraint, medication and electroconvulsive therapy (ECT).

Relatives can declare a patient with a mental disorder to be incapacitous (under the 2002 Civil Procedure Law, number 1/2000) and have the power to make decisions for them in relation to their psychiatric care, finance and social care. These powers are not clearly defined, and there is therefore the potential for decisions that may not be in the person's best interests. The proposed mental health law (Law 29889) aims to clarify this.

People with mental health disorders who lack capacity but need continuing care and who are an ongoing a risk to others are entitled to have a guardian appointed, who will be responsible for their well-being (under the 1984 Civil Code, Legislative Decree 295). If the guardian believes a patient needs admission to hospital, an assessment by two independent psychiatrists (or one physician in an emergency) and judicial authorisation are required.

\section{Treatment provisions under the proposed mental health law}

Law 29889 will clarify and regulate involuntary admissions, emphasising that these have to be for clinical reasons and never for social problems or abandonment by relatives. It states that treatments should be the least restrictive possible, that the necessary psychotropic medications should be available and that hospital conditions should improve.

Patients must be treated in general hospitals, near their homes, and will be referred to a psychiatric hospital only if a particular treatment is unavailable in the general hospital. General hospitals will have to provide a certain number of beds for psychiatric patients and provide treatment in emergencies.

If a patient lacks capacity to provide informed consent for admission, a medical review body comprising at least two psychiatrists will be able to admit him or her for a maximum of 4 days to an acute assessment unit. A different medical tribunal will then determine whether the patient needs a longer stay.

\section{Conclusions}

The Constitution of the Republic of Peru provides a good basis for the development of mental health law in Peru. However, there are currently major gaps in provision, leading to significant human rights breaches. The most important are the lack of a proper procedure to authorise admission, the lack of an appeal process and problems in relation to maximising the potential for people with mental health problems to make decisions for themselves. The Peruvian government is trying to address these issues.

\section{Reference}

American Psychiatric Association (1994) Diagnostic and Statistical Manual of Mental Disorders (4th edn) (DSM-IV). APA. 